\title{
Effect of Moisture, Water Activity and Packaging Materials on Quality and Shelf Life of Some Locally Packed Chanachur
}

\author{
K. Chowdhury*, S. Khan, R. Karim, M. Obaid and G.M.M.A. Hasan \\ Institute of Food Science \& Technology (IFST), BCSIR, Dhaka-1205, Bangladesh.
}

\begin{abstract}
Five different brands of locally produced chanachur packets were collected from the market. From each brand, packets of chanachur having the same date of production were bought and stored at ambient condition $\left(30^{\circ} \mathrm{C}\right.$ and $\left.60 \% \mathrm{RH}\right)$ for 3 months. The changes in water activity, moisture $\%$ and consequent effect on sensory qualities like texture, color, flavor and overall acceptance were investigated every month starting from zero month. Test of packaging materials were also carried out for each brand of packed chanachur. Moisture and water activity values have been increased with time for all the samples (A-E) whereas sensory scores decreased. However, all the chanachur samples except $\mathrm{C}$ were found acceptable under the study period.
\end{abstract}

Key words: Chanachur, Water activity, Sensory quality, Packaging materials, Moisture content, Storage condition, Shelf life.

\section{Introduction}

Chanachur is a unique snack food. The primary raw materials for snacks are cereals, maize, rice, wheat, potato either fresh or dehydrated and nuts (Man and Jones, 1994). Cha nachur consists of a variable mixture of spicy dried ingredients, fried lentils, peanuts, chickpea, flour-noodles, corn, vegetable oil, flaked rice, fried onion, salt and a blend of spices, i.e., coriander, chili and mustard seed. These are ready-to-eat and are shelf stable for six to twelve weeks at normal room temperature (Man and Jones, 1994).

Shelf life is an important property of any food and is of interest to everyone in food chain from producer to consumer. Well planned and conducted consumer acceptability tests in the form of appropriate sensory analysis, is an important part of the shelf life evaluation of any snack product (Man and Jones, 1994). Moisture and water vapor transfer act as key influencing factor for shelf life (Steele, 2004). Physicochemical changes in food during storage can cause loss of shelf-life resulting deterioration of its quality (Ooraikul and Stiles, 1991).

Water is a constituent of food which affects food safety, stability, quality and physical properties (Lewicki, 2004). Increased moisture of food due to water sorption from the atmosphere or by mass transport from neighboring compo-

* Corresponding author: E-mail: kc.ifst@gmail.com nents results in a soggy, soft texture (Lewicki et al, 2004). Snack foods have low moisture content and high fat content and therefore are highly affected by moisture, oxygen and changes in flavor (Fontana Jr., 2008).

Water activity is another critical factor in determining the shelf life of product. Rates of exchange of moisture through the packaging material and the rate of change in water activity of food towards a critical limit will determine the shelf life of a product (Nieuwenhuijzen et al, 2008). Water activity brings a supplement of information as it accounts for the availability of water for degradation reactions (Mathlouthi, 2001). It has become the basic controlling factor in the preservation of foods against microbiological, chemical and physical deterioration (Van Den Berg and Bruin, 1981). Many foods have a water activity level in the range of 0.3 for very dry foods to 0.99 for moist fresh foods (Worobo and Zakour, 1999).

Off-flavors, off-odors and loss of crispiness in packaged food are the major cause of consumer rejection. These might be caused by oxidative rancidity of packaged food, permeation, migration and reactions between packaging components with the food components (Ackermann et al, 1995).

Most food products rely on their particular packaging to achieve their expected shelf life (Steele, 2004). Rates of 
moisture exchange through the package and the rate of change in water activity of the food toward a critical limit determines the shelf life of a product (Fontana Jr., 2008). In modern age, food packaging has become very important because of protection of the product from contamination by macro and microorganisms and their filth, prevention from loss or gain of moisture, shielding the product from oxygen and to facilitate handling (Ball, 1960). Good packaging actually serves two purposes. Technical aspects in packaging aim to extend the shelf life of the food by better protection from all the hazards during storage whereas presentational aspects of packaging increase sales by creating brand image that the buyer usually recognizes and draw attention of better printing quality and glossiness (Peter and Axtell, 1993).

Informations on food packaging in the perspective of Bangladesh are very rarely found. That is why present work was undertaken to evaluate the quality and shelf life of some chanachur from popular companies available in the local market. The aim was to investigate the relation between moisture, water activity and sensory quality such as color, flavor textures etc as well as the packaging materials including storage condition.

\section{Materials and Methods}

\section{Materials}

Five different brands of chanachur from five different wellknown companies were selected as materials of this study. Attempt has been made to select commonly consumed nationally available brand. From each brand, four packed of chanachur having same date of production were purchased from the market and stored under ambient condition for 3months. Five different brands were coded as A, B, C, D, and E. Analyses for water activity, moisture content and sensory evaluations were done at one-month interval starting from zero month during the period between May 2008 to August 2008. All estimations were done in triplicate. Packaging materials of these five brands of chanachur were also studied.

\section{Methods}

\section{a) Test methods of chanachur}

i) Moisture: The moisture content was determined by using an electronic Moisture Analyzer (Sartorius MA-30, Japan). ii) Water Activity $\left(\mathrm{a}_{\mathrm{W}}\right)$ : Water activity was determined by Water Activity Analyzer-TH-500 Aw Sprint, Novasina, Switzerland. The water activity scale extends from 0.0 (bone dry) to 1.0 (pure water). Aw represents the ratio of the water vapor pressure of the food to the water vapor pressure of the pure water under the same conditions and expressed as a fraction. If we multiply the ratio by 100 , we obtain the equilibrium relative humidity $(\mathrm{ERH})$ that the food would produce if enclosed in air in a sealed container at constant temperature. Thus a food with water activity $\left(\mathrm{a}_{\mathrm{W}}\right)$ of 0.7 would produce an ERH of $70 \%$.

III) Sensory Evaluation: Sensory evaluation was carried out every month for each brand of chanachur. A nine point hedonic scale (Peryam \& Pilgrim, 1957) was used to test for flavor, texture, color and overall acceptability of the chanachur.

The degree of likeliness to which a product was expressed as:

$\begin{array}{lc}\text { Like/Dislike } & \text { Points } \\ \text { Like extremely } & 9 \\ \text { Like very much } & 8 \\ \text { Like moderately } & 7 \\ \text { Like slightly } & 6 \\ \text { Neither like nor dislike } & 5 \\ \text { Dislike slightly } & 4 \\ \text { Dislike moderately } & 3 \\ \text { Dislike very much } & 2 \\ \text { Dislike extremely } & 1\end{array}$

A seven member's panel of IFST, BCSIR, has performed sensory evaluation. The same panel members participated in the evaluation session every month starting from zero month. The test was conducted in the laboratory of Oilseed \& Lipid Technology Section, IFST, BCSIR. Five different brands of sample were presented separately to each of the panelists for evaluation. A mean evaluation score of each attribute was repeated for each brand.

\section{b) Packaging Material Test}

i) Thickness: The thickness of the packaging material was measured by electronic thickness gauge/meter, ModelB-1, TOYO SEIKI, LTD, Japan. 
ii) Material Identification: Identification of the packaging material of each brand of chanachur was done by Fourier Transform Infrared Spectrophotometer (FTIR) - model 8400 , Shimadzu, JAPAN by comparing with the reference packaging material of local manufacturer

\section{Results and Discussion}

In the present work, the quality change of the chanachur stored at ambient condition $\left(30{ }^{\circ} \mathrm{C}\right.$ and $\left.60 \% \mathrm{RH}\right)$ for three months were evaluated by monitoring the moisture, water activity, sensory acceptability and packaging material.

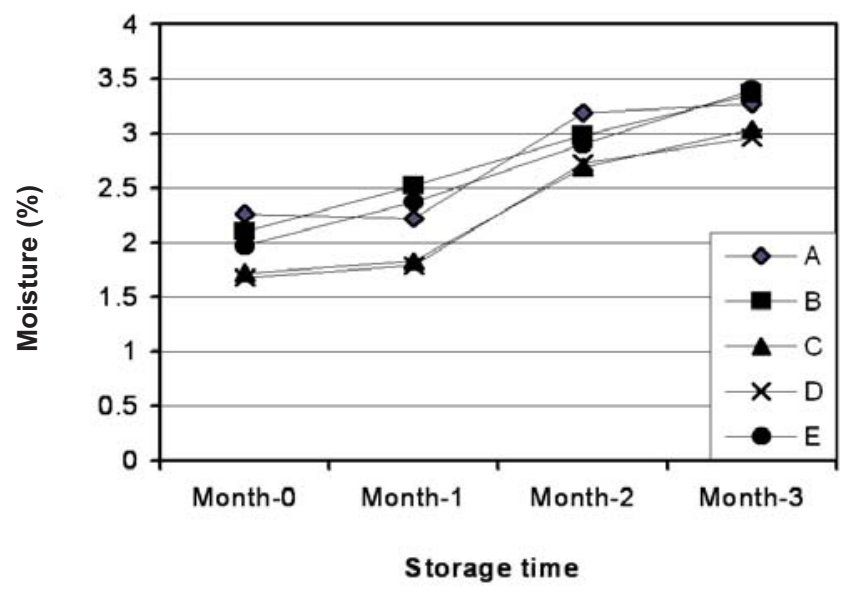

Fig. 1: Changes in moisture content in different brands of chanachur packets (A-E) during storage

\section{Moisture}

Moisture content is the total moisture i.e. bound plus free water present in the sample. Figure 1 shows that initially the moisture content of different brands of chanachur (A-E) are low, ranging from $(1.68-2.26) \%$, whereas chanachur D shows the lowest (1.68) and A shows the highest value (2.26). Chanachur B, C and E have moisture content of 2.1 $\%, 1.72 \%$ and $1.97 \%$ respectively. The moisture content of chanachur within the packet has shown an increasing trend with time. During the first month increase is almost linear ranging from $1.79-2.52 \%$.But during the second month moisture gain is much higher ranging from 2.69-3.19\%. In the third month, the increase is little compared to second month but compared to the initial values $(1.68-2.26 \%)$, the total increase is significant (almost $200 \%$ ). At the end of the study period, moisture $\%$ of $\mathrm{A}=(3.29), \mathrm{B}=(3.36), \mathrm{C}=(3.04)$, $\mathrm{D}=(2.96), \mathrm{E}=(3.4)$. However, the rate of moisture gain from environment is not same for all the packets of chanachur.
Snack foods suffer in quality as a result of moisture gain (Steele, 2004). As chanachur contains oil, it quickly absorbs moisture from the environment and thus results in loss of quality. The findings of increased moisture $\%$ correlate with the sensory evaluation score of the present study. Similar result was observed in the findings obtained by Duizer et al (1998) where they showed that the snacks were perceived to be more crisp with decreased moisture content.

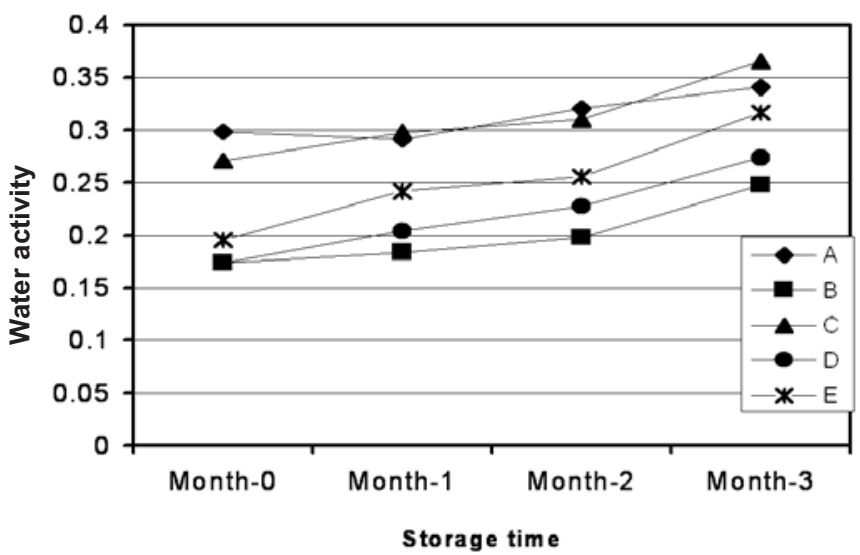

Fig. 2: Changes in water activity in different brands of chanachur packets (A-E) during storage

Water activity: Water activity is the measurement of free moisture and is usually expressed as $" \mathrm{a}_{\mathrm{w}}$ " or percentage Equilibrium Relative Humidity (\% ERH). Figure 2 shows that the changes in water activity values of chanachur packets (A-E) during 3-months storage period. A slightly different scenario exists in the aw value of the packed chanachur. From fig 2 it can be stated that aw of all chanachur samples have been increased in a uniform pattern from zero to next two months. But in the third month, aw of these samples showed a higher ascending rate compared to the previous two months. It reveals that initially aw of chanachur packet A and $C$ showed higher value $0.29867 \& 0.27133$ than the other packets, which are respectively 0.1733 for $\mathrm{B}, 0.17357$ for D \& 0.19533 for E. Finally, the aw of $C=(0.365)>$ $\mathrm{A}=(0.341)>\mathrm{E}=(0.317)>\mathrm{D}=0.274>\mathrm{B}=(0.247)$. This finding indicates that the aw of the chanachur B is within the critical aw range (0.3-0.5) for dry foods (Katz and Labuza, 1981) whereas $\mathrm{C}$ exceeds this limit.

Toledo (1991) stated that if water activity $\left(\mathrm{a}_{\mathrm{w}}\right)$ value is lower than 0.70 it prevents microbiological damage, but to prevent deteriorative chemical and biochemical reactions, it is necessary that the dryness be reduced to a water activity value 
Table I: Sensory Evaluation Scores of different brands of Chanachur stored at ambient condition for 3-months

\begin{tabular}{|c|c|c|c|c|c|c|}
\hline Attributes & Storage month & A & B & $\mathrm{C}$ & $\mathrm{D}$ & E \\
\hline \multirow{5}{*}{ Color } & 0 & $7.00 \pm 1.53$ & $8.14 \pm 0.69$ & $6.57 \pm 1.27$ & $8.00 \pm 0.58$ & $7.14 \pm 1.68$ \\
\hline & 1 & $7.00 \pm 0.58$ & $8.00 \pm 0.82$ & $6.29 \pm 1.70$ & $7.80 \pm 0.79$ & $7.14 \pm 1.07$ \\
\hline & 2 & $6.71 \pm 1.38$ & $8.00 \pm 0.58$ & $6.00 \pm 0.82$ & $7.80 \pm 1.11$ & $7.00 \pm 1.63$ \\
\hline & 3 & $6.57 \pm 0.97$ & $7.86 \pm 0.90$ & $5.71 \pm 1.25$ & $7.60 \pm 1.21$ & $7.00 \pm 1.15$ \\
\hline & 0 & $7.00 \pm 1.15$ & $7.86 \pm 0.69$ & $6.00 \pm 1.15$ & $7.57 \pm 0.97$ & $7.28 \pm 0.95$ \\
\hline \multirow{3}{*}{ Flavor } & 1 & $6.86 \pm 0.90$ & $7.71 \pm 1.50$ & $5.71 \pm 1.50$ & $7.28 \pm 0.99$ & $6.86 \pm 1.34$ \\
\hline & 2 & $6.71 \pm 0.95$ & $7.57 \pm 0.98$ & $5.57 \pm 1.40$ & $7.14 \pm 0.69$ & $6.86 \pm 0.69$ \\
\hline & 3 & $6.28 \pm 1.60$ & $7.57 \pm 1.40$ & $4.43 \pm 0.79$ & $6.86 \pm 1.57$ & $6.86 \pm 0.69$ \\
\hline \multirow{4}{*}{ Texture } & 0 & $6.71 \pm 1.15$ & $8.00 \pm 0.82$ & $6.42 \pm 1.72$ & $8.00 \pm 0.79$ & $8.00 \pm 0.75$ \\
\hline & 1 & $6.43 \pm 1.27$ & $7.86 \pm 1.07$ & $5.85 \pm 1.21$ & $7.86 \pm 1.07$ & $7.57 \pm 0.97$ \\
\hline & 2 & $6.43 \pm 0.97$ & $7.71 \pm 1.11$ & $5.57 \pm 1.40$ & $7.86 \pm 1.21$ & $7.28 \pm 1.11$ \\
\hline & 3 & $6.28 \pm 1.50$ & $7.57 \pm 0.98$ & $5.14 \pm 0.69$ & $7.28 \pm 1.11$ & $7.14 \pm 0.90$ \\
\hline \multirow{4}{*}{ Taste } & 0 & $7.00 \pm 1.52$ & $7.86 \pm 0.69$ & $5.71 \pm 1.50$ & $7.28 \pm 1.25$ & $7.00 \pm 1.00$ \\
\hline & 1 & $6.86 \pm 1.07$ & $7.86 \pm 1.07$ & $5.57 \pm 0.97$ & $7.57 \pm 0.97$ & $6.86 \pm 1.07$ \\
\hline & 2 & $6.57 \pm 1.27$ & $7.85 \pm 1.07$ & $5.28 \pm 1.11$ & $7.43 \pm 1.27$ & $6.86 \pm 1.57$ \\
\hline & 3 & $6.57 \pm 1.51$ & $7.71 \pm 1.11$ & $4.43 \pm 0.97$ & $6.71 \pm 0.75$ & $6.86 \pm 1.11$ \\
\hline \multirow{4}{*}{$\begin{array}{l}\text { Overall } \\
\text { acceptance }\end{array}$} & 0 & $7.00 \pm 1.52$ & $8.00 \pm 1.00$ & $5.85 \pm 1.34$ & $7.71 \pm 0.95$ & $7.28 \pm 0.75$ \\
\hline & 1 & $6.86 \pm 1.07$ & $7.86 \pm 1.07$ & $5.58 \pm 1.34$ & $7.43 \pm 0.79$ & $7.00 \pm 1.00$ \\
\hline & 2 & $6.71 \pm 1.25$ & $7.86 \pm 1.07$ & $5.71 \pm 1.11$ & $7.28 \pm 1.25$ & $7.00 \pm 1.00$ \\
\hline & 3 & $6.71 \pm 1.25$ & $7.75 \pm 0.70$ & $4.43 \pm 0.97$ & $6.86 \pm 0.90$ & $6.86 \pm 1.57$ \\
\hline
\end{tabular}

Scores are based on a 9 point hedonic scale where Score of $9=$ Like extremely $5=$ neither like or dislike $1=$ Dislike extremely.

lower than 0.3. (Roudaut et al, 1998, 2000). Sensory Analysis: Table I reveals the detailed sensory analysis of the packed chanachur (A-E) during 3 months period. Interestingly, although all chanachur packets have similar date of production, the initial quality differs much.

Observing the texture attributes and crispness of the stored chanachur packet in Table I, it reveals that the initial value of $\mathrm{A}$ and $\mathrm{C}$ are poor in $6.71 \pm 1.15$ and $6.42 \pm 1.72$ compared to those B, D, and E (Nearly 8 for all). This corresponding to the high initial aw value of these two brands of chanachur ( 0.29867 for $A$ and 0.27133 for $C$ ). The initial taste score of chanachur $\mathrm{C}$ is also very poor $(5.71 \pm 1.50)$. At zero month, overall acceptance score of chanachur $\mathrm{C}$ is also very low $(5.85 \pm 1.34)$. Chanachur $\mathrm{A}$ and $\mathrm{E}$ is of intermediate quality (Acceptance score $7.00 \pm 1.52$ and $7.28 \pm 0.75$ respectively) whereas chanachur B and D are of very sound quality (acceptance score $8.00 \pm 1.00$ and $7.71 \pm 0.95$ respectively). At the end of study period (i.e. after 3 months storage), overall acceptance score of samples (A-E) are $\mathrm{A}=6.71 \pm 1.25, \mathrm{~B}=$ $7.75 \pm 0.70, \mathrm{C}=4.43 \pm 0.97, \mathrm{D}=6.68 \pm 0.90$ and $\mathrm{E}=6.68 \pm$ 1.57 which indicates the lowest score of $\mathrm{C}$.

These results are similar to those obtained by Katz and Labuza (1981), Seymour and Hamann (1988) and Sauvageot and Blond (1991). Each of these researchers, studying different types of crisp foods, determined that as the water activity

Table II: Study of Packaging Material

\begin{tabular}{c|c}
\hline Sample Code & Thickness \\
\hline A & $0.0673 \pm 0.0005$ \\
B & $0.0650 \pm 0.001$ \\
C & $0.0763 \pm 0.001$ \\
D & $0.0713 \pm 0.001$ \\
E & $0.0636 \pm 0.0005$ \\
\hline
\end{tabular}




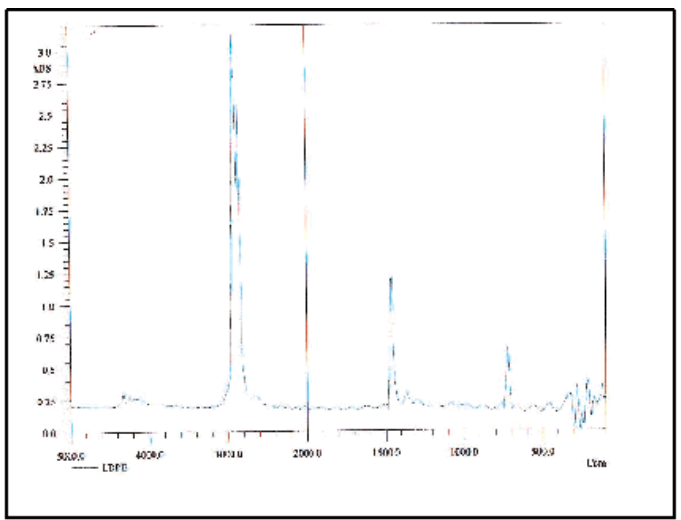

(a)

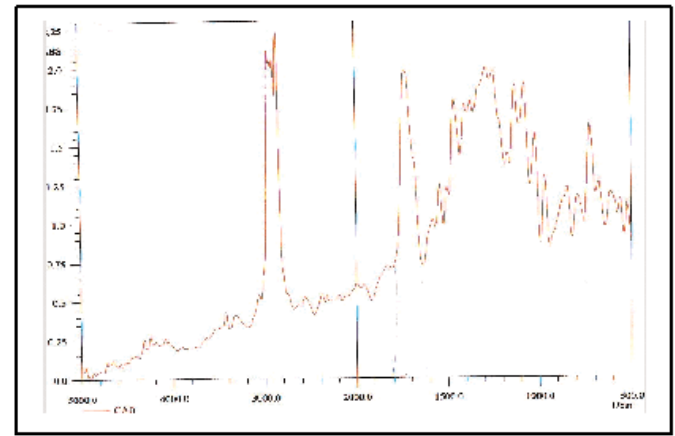

(c)

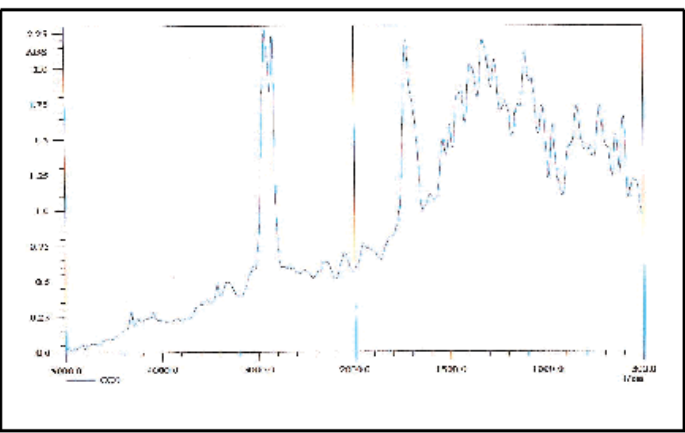

(e)

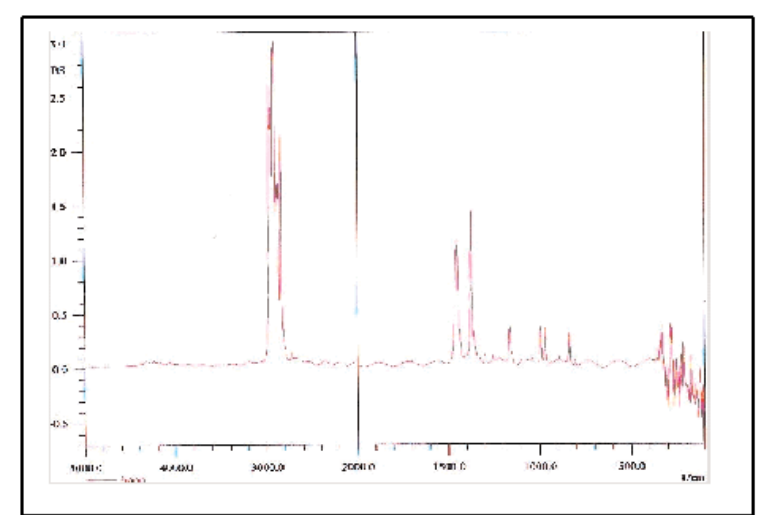

(b)

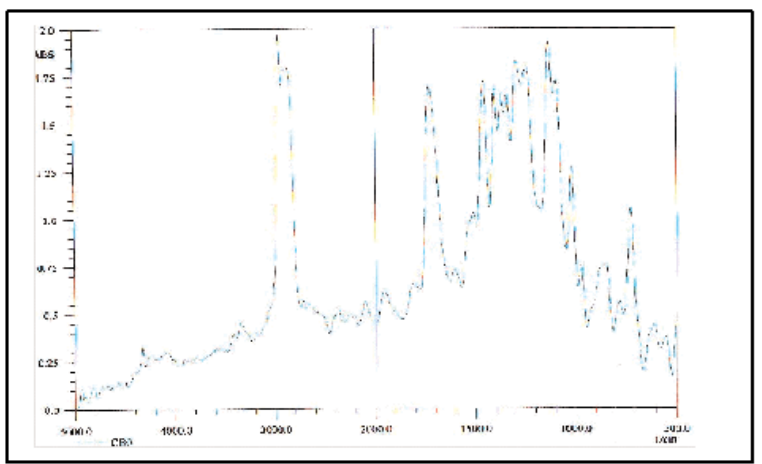

(d)

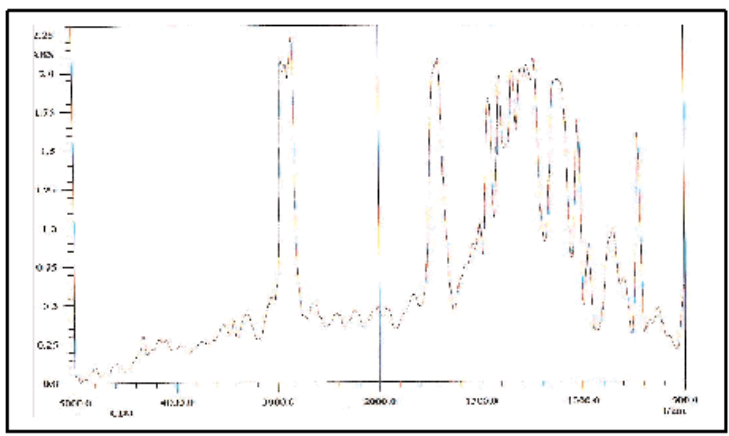

(f)

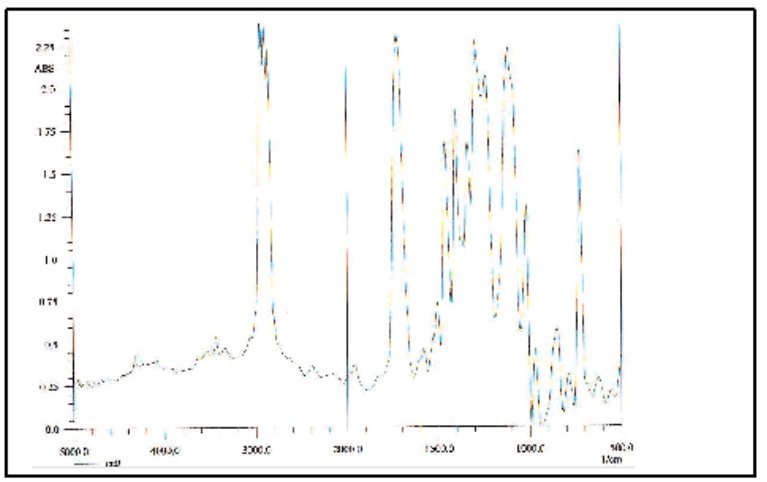

(g)

Fig. 3: FTIR Spectra of Reference Packaging Materials of Local Manufacturer - (a) LDPE, (b) BOPP and Packaging Materials of 5 Brands of Chanachur - (c) CA0, (d) CB0, (e) CC0, (f) CD0 and (g) CE0 
is increased, sensory crispness is decreased. Critical water activity $\left(\mathrm{a}_{\mathrm{w}}\right)$ where the products become organoleptically unacceptable generally fall in the $0.35-0.50$ aw range (Katz and Labuza, 1981). In sample C, with initial water activity level was $\left(\mathrm{a}_{\mathrm{w}}\right) 0.27133$, lost its crispiness when it reached to 0.365 at the end of the storage period.

Therefore, during storage period the taste as well as the overall quality of the chanachur $\mathrm{C}$ is deteriorated \& finally becomes unacceptable from the sensory standpoint. Chanachur A, B, D, E have been found acceptable where B is of superior quality.

\section{Packaging Materials}

Packaging material study has been carried out to observe the effects of packaging materials on the stability of chanachur during ambient storage. Table II reveals thickness property of the packaging materials used for chanachur packaging. Thickness of those packaging materials ranges between 0.063-0.0763 millimeters. Statistically, thickness variation of all the packaging materials is not significant. It shows that they are mostly uniform.

Snack foods are typically packaged in multi-layer structures (Robertson, 1998). FTIR spectrum study of packaging materials of five brands of chanachur was done. IR spectrum of functional groups of each packaging material is unique. Packaging materials are polymer compounds. An organic compound is identified by finding a reference or transmittance IR spectrum that matches that of the unknown compound (Settle, 1997). After observing FTIR spectrum of all the five packaging materials together, they were found almost identical. From figure 3 the first IR absorption in these materials was found in $2800-3300 \mathrm{~cm}^{-1}$ absorbance region which was expected to consist solely of methylene stretches and bends (C-H stretching region) of polyethylene (Shimadzu, 2001). This stretch matches with a stretch in 2780-3150 $\mathrm{cm}^{-1}$ absorbance region of LDPE (Low density polyethylene) and BOPP (Biaxially oriented polypropylene) as per manufacturer packaging material specification used as standard reference material. The $1950-1450 \mathrm{~cm}^{-1}$ absorbance region exhibits IR absorption from a wide variety of double bonded functional groups. Below $1500 \mathrm{~cm}^{-1}$ absorbance region is termed as the fingerprint region of characteristic functional groups (Shimadzu, 2001). Due to crystalinity of
LDPE, the split and additional peaks in the absorbance region 1464 and $719 \mathrm{~cm}^{-1}$ were observed (O'Keefe, 2004). From all these studies of stretching regions it can be concluded that the chanachur packaging materials of these five brands of chanachur might be composed of BOPP, vacuum coated with aluminum and later on laminated with thermosetting LDPE in the inner side (BOPP/Al/LDPE). External side of BOPP is colorfully printed distinct for each brand. Similar study was observed in Robertson (1998) where potato chips packaged in PP/aluminum foil pouches required about 27 weeks to become unsalable because rancid flavors developed.

Labeling of chanachur packet $\mathrm{C}$ shows that $\mathrm{N}_{2}$ flush was done to keep extra freshness of the product. The other four chanachur packets were not labeled so. But sensory evaluation score indicated chanachur $\mathrm{C}$ as the worst one among five. This gives an idea that either $\mathrm{N}_{2}$ flush has not been optimized or is not appropriate in packaging to maintain the quality of chanachur during shelf life storage.

\section{Conclusion}

The study focuses on the crucial evaluations required to extend shelf life of processed snack food like chanachur. Shelf life of chanachur also depends on the initial quality and hygiene of the raw materials used. Results showed there is a correlation between aw, sensory quality (crispiness, texture, flavor), moisture percent and packaging technology for shelf life of the studied samples. Chanachur B having water activity $(0.173)$ and moisture content $(2.1 \%)$ with sensory overall acceptance score $(7.75 \pm 0.70)$ was proved to be the appropriate one to extend the shelf life. Snack foods can be successfully stored at room temperature by proper control of their water activity $\left(\mathrm{a}_{\mathrm{w}}\right)$ and packaged by appropriate packaging material.

\section{Acknowledgement}

This paper is an outcome of R \& D project work "Studies on the Packaging Materials used in Different Bakery Products (Bread, Biscuit \& Chanachur) of the Local Confectionaries in the light of HACCP" of IFST, BCSIR, Dhaka. The instruments used in this work have been supported by an ADP project funded by the Ministry of Science and Information and Communication Technology, Government 
of Bangladesh. We are grateful to the BCSIR authorities for providing the scope to conduct the study and to Mr. Abdul Latif, Ex-Chief Scientific Officer, IFST \& Dr. Mamtaz Dowlatana, Director, BCSIR. Both of them were Project Directors of the above-mentioned ADP project. We are also grateful to Dr. Barun Kanti Saha, SSO and Abdur Rahim, JEO, IFST. Both were sensory test panelists of this work.

\section{References}

Ackermann P., Jagerstad M. and Ohlsson T (1995). Foods and Packaging Materials - Chemical Interactions. 1st Ed. (The Royal Society of Chemistry, UK), pp 33, 34, $65,137$.

Ball C. O. (1960). Here are effects on color changes in packaging of fresh meat cuts. Natl. Provisional 143(47): 10 $-12$.

Duizer L. M., Campanella O. H. and Barnes G. R. G. (1998). Sensory, instrumental and acoustic characteristics of extruded snack food products. J.Text.Stud. 29: 397411.

Fontana Jr., A.J. (2008). Understanding the importance of water activity in food. Inn. Food Tech. pp 116 - 117

Katz E. E and Labuza T. P. (1981). Effect of Water Activity on the Sensory Crispness and Mechanical Deformation of Snack Food Products. J. Food Sci., 46: 403-409.

Lewicki P. P. (2004) Water as the determinant of food engineering properties: A review. J. Food Eng., 61: 483 495.

Lewicki P. P., Jakubczyk E., Marzec A., Cabral M. C. and Pereira P.M. (2004) Effect of water activity on mechanical properties of dry cereal products. Acta Agro. 4(2): 381 - 391.

Man C. M. D. and Jones A. A. (1994). Shelf Life Evaluation of Foods. 1st Ed. (Blackie Academic \& Professional, London) pp 202-203.

Mathlouthi M. (2001). Water content, water activity, water structure and the stability of foodstuffs. Food Control, 12:409 - 417.
Nieuwenhuijzen N. P. V., Martin C. P, Meinders M. B. J., Tromp R. H., Hamer R. J. and Vile T. V. (2008). Water Content or Water Activity: What Rules Crispy Behavior in Bread Crust? J. Agric. Food Chem., 56(15): 6432-6438.

O'Keefe J. F. (2004). Identification of Polymers by IR Spectroscopy. Rubber World, pp 1-6.

Ooraikul B. and Stiles M. E (1991). Modified Atmosphere Packaging of Food. 1st Ed. (ELLIES HORWOOI, London) pp $28 \& 30$.

Peryam D. R. and F.J. Pilgrim (1957). Hedonic scale Method of measuring food preference. Food Technol. II: 9-14 (suppl.).

Peter F. and Axtell B. (1993). Appropriate Food Packaging, Publ. Transfer of Technology for Development. Amsterdam, International Labor office, Geneva.

Robertson G. L. (1998). Food Packaging Principles and Practice (Marcel Dekker, Inc., New York) pp 575

Roudaut G., Dacremont C., Le Meste M., Pamies B.V. and Mitchell J.R., (2000). Understanding the texture of Low Moisture Cereal Products: Mechanical and Sensory Measurements of Crispness. J. Sci. and Food Agri., 80 pp 1679-1685

Roudaut G., Dacremont C. and Le Meste M., Pamies B. V. and Mitchell J. R. (1998). Influence of water on the Crispness of cereal-Based foods: Acoustic, Mechanical and Sensory Studies. J. Tex. Stud., 29(2) pp 199-213.

Sauvageot F. and Blond G. (1991). Effect of water activity on crispness of breakfast cereals. J.Text.Stud. 22: 423 429.

Settle F. S. (1997). Handbook of Instrumental Techniques for Analytical Chemistry, 2nd ed., (Prentice Hall, New Jersey) pp 270.

Seymour, S. K. and Hamann D. D. (1988) Crispness and crunchiness of selected low moisture foods. J. Text. Stud. 19: 79 - 86.

Shimadzu (2001). General Operation Guide for Shimadzu FTIR 8000 Series (FTIR-8400/8900) pp 21, 22 
Steele R. (ed.) (2004). Understanding and measuring the shelf-life of food. Woodhead Publishing Ltd. Cambridge. U.K.

Toledo R. T. (1991). Fundamentals of Food Process Engineering, 2nd ed., Chapman \& Hall, New York (NY), USA, pp. 456-506.
Van Den Berg C. and Bruin S. (1981). Water activity and its estimation in food: Systems Theoretical aspects. In "Water Activity: Influences on Food Quality." ed. By L.B. Rockland and G.F. Stewart. Academic Press, London, pp.4-8.

Worobo R. and Zakour O. P. (1999). Water activity: another critical factor for safety of food products. Venture. 1(4): $1-5$.

Received: March 04, 2010;

Accepted: March 30, 2010 